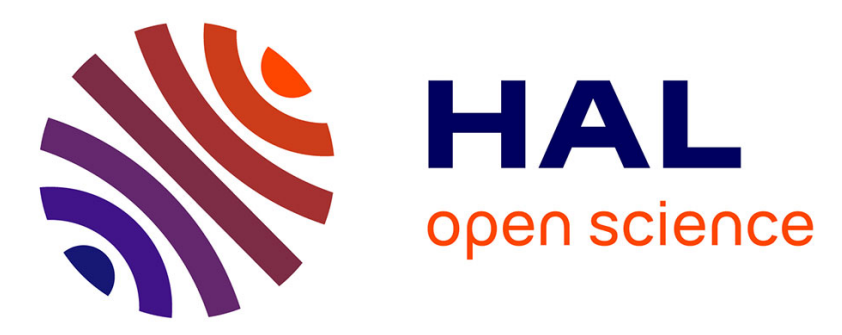

\title{
Générateur digital de champ tournant pour l'observation de la propagation des domaines magnétiques cylindriques
}

\author{
G. Bonnes, H. Jouve, Damien Mauduit
}

\section{> To cite this version:}

G. Bonnes, H. Jouve, Damien Mauduit. Générateur digital de champ tournant pour l'observation de la propagation des domaines magnétiques cylindriques. Revue de Physique Appliquée, 1974, 9 (2), pp.501-502. 10.1051/rphysap:0197400902050100 . jpa-00243809

\section{HAL Id: jpa-00243809 https://hal.science/jpa-00243809}

Submitted on 1 Jan 1974

HAL is a multi-disciplinary open access archive for the deposit and dissemination of scientific research documents, whether they are published or not. The documents may come from teaching and research institutions in France or abroad, or from public or private research centers.
L'archive ouverte pluridisciplinaire HAL, est destinée au dépôt et à la diffusion de documents scientifiques de niveau recherche, publiés ou non, émanant des établissements d'enseignement et de recherche français ou étrangers, des laboratoires publics ou privés. 


\title{
GÉNÉRATEUR DIGITAL DE CHAMP TOURNANT POUR L'OBSERVATION DE LA PROPAGATION DES DOMAINES MAGNÉTIQUES CYLINDRIQUES
}

\author{
G. BONNES, H. JOUVE et D. MAUDUIT
}

Laboratoire d'Electronique et de Technologie de l'Informatique, Centre d'Etudes Nucléaires BP 85, 38041 Grenoble-Cedex, France

(Reçu le 20 juillet 1973, révisé le 9 novembre 1973)

\begin{abstract}
Résumé - La synthèse digitale de 2 sinusoïdes en quadrature de phase permet l'observation du déplacement de domaines magnétiques cylindriques, depuis les très basses fréquences jusqu'à $50 \mathrm{kHz}$.
\end{abstract}

Abstract. - The digital synthesis of two sine waves $90^{\circ}$ out of phase makes possible the observation of the motion of cylindrical magnetic domains (" magnetic bubbles ») from very low frequencies up to $50 \mathrm{kHz}$.

Les domaines magnétiques cylindriques ou bulles [1] qui existent dans les lames minces d'orthoferrites de terres rares ou de grenats substitués sont très étudiés en raison de leur possibilité d'utilisation comme mémoire magnétique.

Le déplacement des bulles (mémoires circulantes) est obtenu par des motifs de permalloy aimantés à l'aide d'un champ magnétique tournant parallèle au plan de la lame.

Une étude précise des phénomènes de propagation des bulles requiert la possibilité de les immobiliser ou de les observer en un endroit quelconque de leur parcours, ceci dans une gamme de fréquences aussi large que possible.

Nous avons donc été conduits à définir un générateur de champ magnétique tournant dont les caractéristiques sont les suivantes:

- Fréquence de rotation variable de 0 à $5 \times 10^{4} \mathrm{~Hz}$.

- Possibilités d'arrêter à tout moment le champ dans une direction choisie et d'inverser le sens de rotation.

- Stroboscopie avec fenêtre d'observation variable et visualisation en position fixe ou en défilement à vitesse lente.

Le champ magnétique est créé par deux paires de bobines de Helmholtz alimentées en quadrature de phase.

Alors que les appareils décrits par d'autres auteurs [2], [3] utilisent des systèmes analogique (sans possibilité d'arrêt) ou mécanique (limité aux très basses fréquences), le choix de la synthèse digitale permet à la fois l'arrêt sur une position déterminée et des déphasages variables, indépendants de la fréquence.

Les pièces maîtresses de ce générateur (Fig. 1) sont 3 compteurs-décompteurs $(\mathrm{CD})$ à 5 digits qui définissent ainsi 62 états. Les 2 premiers le $C D$ pilote et le $\mathrm{CD}$ asservi servent à la génération des 2 courants sinusoïdaux alimentant les bobines, le troisième sert à l'affichage des positions.

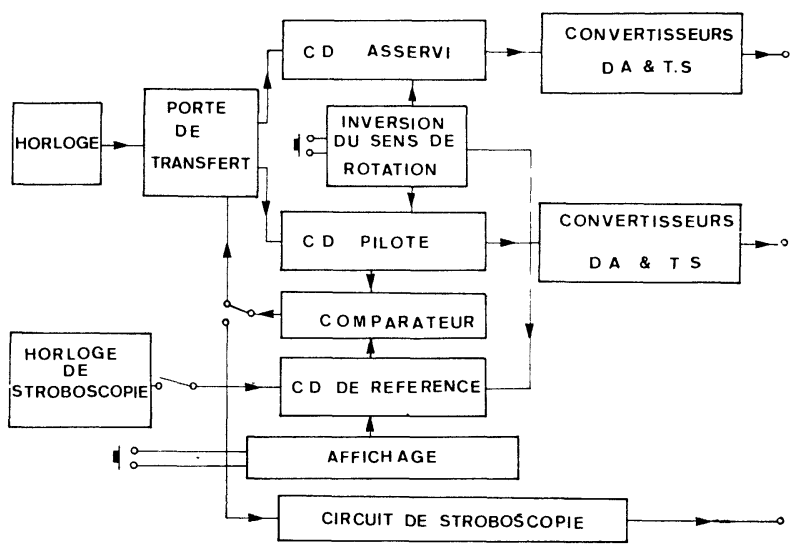

Fig. 1. - Composition d'ensemble du générateur de signaux électriques.

Une horloge pilote de fréquence réglable commande alternativement, par l'intermédiaire d'une bascule bi-stable, le $C D$ pilote et le $C D$ asservi. Le déphasage entre les 2 voies est maintenu grâce à des " tops " de synchronisation déclenchés par chaque passage 
de CD pilote dans l'état (00 000) qui 'impose alors au CD asservi l'état (00 111) ou (11 000) selon le sens de rotation. La partie inversion du sens de rotation (Fig. 1), effectue les opérations d'inversion du sens de comptage et de passage à l'état complémentaire pour les circuits de synchronisation.

Ensuite, un ensemble de convertisseurs digitauxanalogiques puis triangulaires-sinusoïdaux transforment les niveaux logiques ainsi créés en sinusoïdes.

L'arrêt du champ tournant se fait en comparant l'état du CD pilote avec un état affiché au niveau du $\mathrm{CD}$ de référence.

Le $\mathrm{CD}$ de référence sert aussi à positionner 1a fenêtre de stroboscopie. En observation fixe on affiche la valeur du début de cette fenêtre. Un circuit compare les états du $C D$ pilote et du $C D$ de référence pour envoyer, au moment de la coïncidence, une impulsion électrique qui déclenche le laser.

La durée de cette impulsion, fixée à l'aide de compteurs décimaux, peut varier de 0 à 100/124e de période.

Le déplacement de la fenêtre d'interrogation est obtenu en pilotant le $\mathrm{CD}$ de référence par une horloge à la fréquence de $62 \mathrm{~Hz}$, ce qui réalise un défilement à la fréquence de $1 \mathrm{~Hz}$.

Pour illustrer les possibilités de cet appareil, nous avons relevé les courbes de déplacement de domaines en fonctionnement quasi statique et à $50 \mathrm{kHz}$. Les observations se font en pointant la position du bord avant et du bord arrière du domaine magnétique, visualisé par effet Faraday. Les distances sont repérées par rapport aux motifs de propagation (Fig. 2).

L'examen de ces courbes suggère plusieurs remarques. En premier lieu, il n'y a pas beaucoup de différence entre le mouvement en $\mathrm{TBF}$ et celui à $50 \mathrm{kHz}$, ce qui signifie que même à $50 \mathrm{kHz}$ la bulle a le temps de se mettre en état d'équilibre statique.

On en déduit que $50 \mathrm{kHz}$ est loin d'être la fréquence maximum au-delà de laquelle ne pouvant plus suivre le gradient de champ magnétique qui l'entraîne, la bulle se trouve hors d'état d'équilibre [4] et disparaît.

Un deuxième point important est l'aspect en « marches d'escalier » de la propagation des bulles, et la variation de leurs dimensions.

Cette allure peut s'expliquer d'une part, par les intervalles entre les éléments de propagation, d'autre part par leur configuration qui donne lieu à une anisotropie magnétique de forme. Cette progression irrégulière diminue certainement la fréquence maximum de fonctionnement d'un dispositif à bulles [4].

Les informations obtenues par ces observations sont très précieuses pour la compréhension des phénomènes mis en jeu lors du déplacement, ainsi que pour l'amélioration des performances des circuits de propagation.
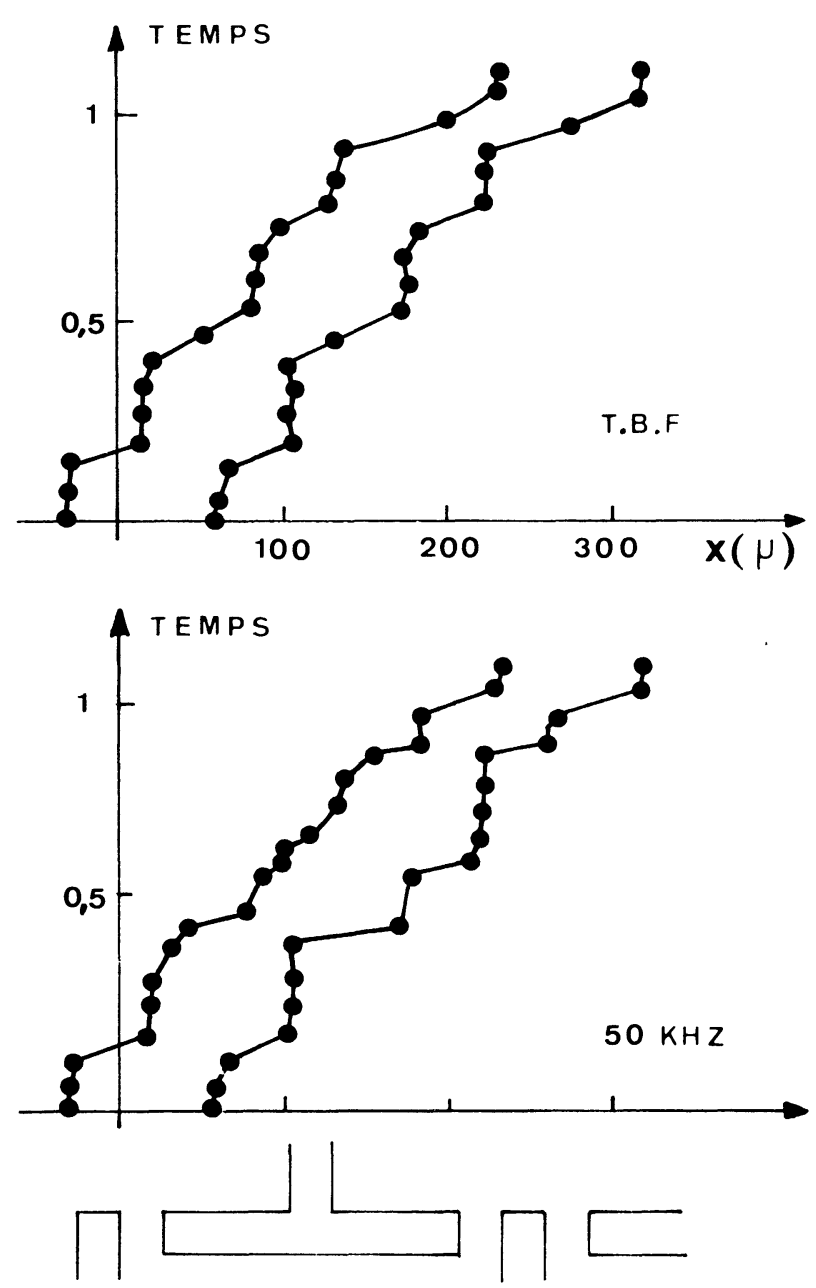

Fig. 2. - Déplacement d'une bulle sur un circuit en fonction du temps. Pour ce dernier on a pris comme unité sur l'échelle verticale une période. Les courbes sont tracées en relevant les positions du bord avant et du bord arrière de la bulle en fonction de la position du champ tournant. Le tracé en TBF est obtenu, au " coup par coup ", le tracé à $50 \mathrm{kHz}$ en stroboscopie fixe. La largeur des motifs qu'on a schématisés sous les courbes est de $26 \mu$, le diamètre de la bulle est de l'ordre de $100 \mu$.

\section{Bibliographie}

[1] Boвeck, A. H., Bell. Syst. Tech. J. 49 (1967) 1901.

[2] Smith, A. B. and Johnson, G. L., Rev. Sci. Instrum. 43 (1972) 1831
[3] Rossol, F. C., IEEE trans. Magn. MAG 7 (1971) 142.

[4] Chen, Y. S., Nelson, T. J., J. Appl. Phys. 44 (1973) 3306. 